\title{
A Medium Access Control Mechanism for Distributed In-band Full-Duplex Wireless Networks
}

\author{
Haiwei Zuo ${ }^{1}$, Yanjing Sun ${ }^{1, *}$, Song $\mathrm{Li}^{1}$, Qiang $\mathrm{Ni}^{2}$, Xiaolin Wang ${ }^{1}$ and Xiaoguang Zhang ${ }^{1}$ \\ ${ }^{1}$ School of Information and Control Engineering, China University of Mining and Technology \\ Xuzhou, Jiangsu 221116 - China \\ [e-mail: yjsun@cumt.edu.cn] \\ ${ }^{2}$ School of Computing and Communications, InfoLab21, Lancaster University, Lancaster, LA1 4WA - U.K. \\ [e-mail: q.ni@lancaster.ac.uk] \\ *Corresponding author: Yanjing Sun
}

Received February 20, 2017; revised April 20, 2017; revised June 11, 2017; accepted July 5, 2017;

published November 30, 2017

\begin{abstract}
In-band full-duplex (IBFD) wireless communication supports symmetric dual transmission between two nodes and asymmetric dual transmission among three nodes, which allows improved throughput for distributed IBFD wireless networks. However, inter-node interference (INI) can affect desired packet reception in the downlink of three-node topology. The current Half-duplex (HD) medium access control (MAC) mechanism RTS/CTS is unable to establish an asymmetric dual link and consequently to suppress INI. In this paper, we propose a medium access control mechanism for use in distributed IBFD wireless networks, FD-DMAC (Full-Duplex Distributed MAC). In this approach, communication nodes only require single channel access to establish symmetric or asymmetric dual link, and we fully consider the two transmission modes of asymmetric dual link. Through FD-DMAC medium access, the neighbors of communication nodes can clearly know network transmission status, which will provide other opportunities of asymmetric IBFD dual communication and solve hidden node problem. Additionally, we leverage FD-DMAC to transmit received power information. This approach can assist communication nodes to adjust transmit powers and suppress INI. Finally, we give a theoretical analysis of network performance using a discrete-time Markov model. The numerical results show that FD-DMAC achieves a significant improvement over RTS/CTS in terms of throughput and delay.
\end{abstract}

Keywords: In-band Full-duplex, Distributed mechanism, Medium access control, Throughput, Delay 


\section{Introduction}

IBFD wireless communication allows nodes to transmit and receive simultaneously in the same frequency band, which can theoretically double the spectral efficiency of half-duplex (HD) wireless communication [1-3]. The ability to suppress self-interference below the noise floor that does not interfere with desired signal reception has been achieved [4, 5], supporting a single node to transmit and receive in IBFD mode. However, for IBFD communication among multiple nodes, the inter-node interference (INI) suppression and MAC protocol must be fully considered for IBFD wireless networking.

In this work, we study the distributed IBFD wireless network lacking a central unit as an access point (AP). All nodes randomly distribute in the network support IBFD wireless communication and have equal access priority. The multi-node IBFD transmission link can be divided into a symmetric dual link between two nodes or an asymmetric dual link among three nodes. Goyal et al. [6] defined these two links and their transmitters/receivers. If the transmitter of uplink is exposed to the receiver of downlink in an asymmetric dual link, the sending signal of uplink will serve as the INI signal of downlink, which negatively affects the desired signal reception. Therefore, to realize asymmetric dual communication in the IBFD wireless networks, it is necessary to determine effective INI suppression methods for design of MAC communication protocols.

Current IBFD INI suppression methods are mainly used in centralized wireless networks, and very few can be leveraged in distributed wireless networks. For a centralized network, the AP node can be used to configure and manage the signal to interference plus noise ratio (SINR) of station nodes using power control, which guarantees a station can receive the desired packet of multiple received packets [7-9] However, in a distributed network, the identity and transmit power of communication nodes in the dual transmission links are indeterminate information for the network. It is difficult to leverage a certain node to calculate SINR and control transmit power. Considering this difficulty, some studies ignored the impact of INI or assumed that INI was successfully suppressed [6, 10, 11]. But these research results cannot be used in the Wireless open-Access Research Platform (WARP) [12, 13] or Universal Software Radio Peripheral (USRP) [14, 15] hardware platform to assess practicability, thus limiting the design and application of distributed IBFD MAC protocols.

To achieve IBFD communication between multiple nodes, MAC communication protocols are required $[3,16]$. HD protocols such as RTS/CTS (Request-to-Send/Clear-to-Send) and CSMA/CA (Carrier Sense Multiple Access with Collision Avoidance) are currently used, but direct incorporation of un-modified versions of these protocols into IBFD wireless communication would face some problems: 1) The network nodes are unable to recognize the transmission mode of communication nodes and determine whether to contend the opportunity of asymmetric IBFD dual communication, which limits total network throughput. 2) They can only assist to establish symmetric dual link but cannot reserve channel for the downlink of asymmetric dual transmission. 3) If the destination node of RTS frame has no packet to transmit or has a packet to another node but not to the source node of RTS, then the IBFD wireless communication with RTS/CTS will work equivalently to HD wireless communication.

In IBFD dual communication, the establishment of an asymmetric dual link is more difficult than that of a symmetric dual link. The asymmetric dual communication must 
consider the method of INI suppression, but also must be able to reserve the uplink and downlink simultaneously by medium access. The 802.11 RTS/CTS mechanism requires two channel accesses to establish an asymmetric dual link, a procedure that is complex and time-consuming. Although some current IBFD MAC protocols [17-19] improved the throughput of symmetric IBFD dual transmission, they are unable to be used in the networks where both synchronous and asynchronous dual communication coexist, precluding exploitation of the full potential of IBFD wireless communication.

To achieve maximum performance gain in practical IBFD wireless networks, we fully consider the existence of INI and propose a new distributed medium access control mechanism, named as FD-DMAC (Full-Duplex Distributed MAC). To address the problems mentioned above, this work makes the following contributions.

- To suppress the INI in asymmetric dual link, we add the received power information of communication nodes in control frame. The transmitter of uplink and downlink can obtain this information by medium access, and then estimate SINR and adjust transmit power, which will minimize the impact of INI during data transmission.

- Based on three-way handshaking, we design a FD-DMAC mechanism that can establish symmetric or asymmetric dual link by single channel access. This mechanism fully supports the two transmission modes of asymmetric IBFD dual communication, in contrast to the studies of [10], [20] and [21] that only considered one transmission mode.

- We assist the communication nodes and their neighbors to precisely know channel transmission status by FD-DMAC medium access. If the communication nodes do not transmit in IBFD mode, the neighbors will contend the opportunity of asymmetric dual communication. This improvement will extend IBFD capability and avoid collisions caused by hidden nodes.

- To characterize the FD-DMAC mechanism and analyze its performance, we derive a Markov model to determine the throughput and delay of IBFD wireless networks, while considering the effect of the two asymmetric dual transmission modes on the performance metric.

The remainder of this paper is organized as follows. Section 2 provides the related literature and Section 3 presents the system model for an IBFD wireless network. Section 4 describes the specific FD-DMAC mechanism for symmetric/asymmetric IBFD dual transmission. Section 5 presents the theoretical analysis of our proposed mechanism by leveraging a Markov model. Section 6 includes the numerical results of FD-DMAC, RTS/FCTS [10] and RTS/CTS mechanism. Section 7 concludes our paper.

\section{Related Works}

It is essential to suppress INI before IBFD dual communication between multiple nodes. Bai et al. [22] proposed a bin-and-cancel INI suppression scheme that utilized Han-Kobayashi style common-private message splitting to divide the uplink transmit signal into two parts. Only IBFD AP can receive and recreate these two parts into the full original signal and the receiving station of the downlink cannot, which will lessen INI. Tang et al. [7] leveraged capture effect to suppress INI in centralized wireless networks. It set the transmit power of AP to be stronger than those of the stations. When AP and two stations communicate in an asymmetric dual transmission, the station can still receive the desired signal from several received signals by capture effect as long as the SINR of the signal from AP is higher than that of the signals from other stations. The methods above both require use of an AP node to 
globally control the stations, and thus these methods can only be applied in centralized wireless networks. INI suppression remains difficult for asymmetric dual transmission in a distributed IBFD wireless network.

Most current IBFD MAC protocols are designed based on standard 802.11 protocols, such as CSMA/CA and RTS/CTS [10, 11, 19, 23-25]. Sen et al. [23] proposed a CSMA/CN (collision notification) protocol. They added a signature between the preamble and the headers of data frame from source node that can only be read by the destination node of the data frame. Once a collision occurs, the destination node detects the unique signature and returns it to the source node, which will abort the dual transmission immediately. Doost-Mohammady et al. [19] directly applied CSMA/CA into IBFD wireless communication. When the destination AP or station node receives the packet header successfully, it transmits its own packet to the source node immediately. If there is no packet to return, it sends out a busytone for channel reservation. However, the MAC protocols proposed in [19] and [23] are only suitable for symmetric IBFD dual transmission and are unable to establish an asymmetric dual link.

For distributed IBFD wireless network, Goyal et al. [6] proposed a MAC protocol without medium access. They designed IBFD transmission flag fields to notify the network nodes of the communication status. However, this scheme may have difficulties in selection of the proper signal and face high collision problems in source-based asymmetric dual transmission since the secondary link is not reserved. Cheng et al. [10] proposed a RTS/FCTS (Full-duplex CTS) mechanism for IBFD wireless networks. It uses one RTS frame and two FCTS frames to complete three-way handshaking of the symmetric or asymmetric dual link. But the length of FCTS is so much longer than that of CTS, which limits the increase of the IBFD network throughput. Moreover, this scheme did not fully consider the two transmission modes of asymmetric IBFD dual transmission, limiting the improvement of network throughput. Furthermore, [6] and [10] ignored the impact of INI during MAC protocol design. Based on [26], Thilina et al. [16] discussed three key mechanisms that can be utilized in the design of IBFD MAC protocols: shared random backoff, header snooping, and collision avoidance with RTS/CTS exchange. To address these points, they proposed three corresponding MAC protocols, but their network model contains only three nodes without consideration of neighbor impact on communication nodes.

\section{System model}

In the IBFD wireless network, a pre-specified number of nodes always have equal access priority and support IBFD wireless communication. The MAC layer is managed by a state machine which follows the same DIFS (Distributed Inter Frame Space), SIFS (Short Inter Frame Space), post-backoff, backoff scheme and medium access mode. We classified the IBFD transmission modes in WLAN [6] as HD transmission mode, symmetric IBFD (SFD) dual transmission mode, and asymmetric IBFD (AFD) dual transmission mode. AFD can be further classified as destination-based asymmetric IBFD (DAFD) dual transmission mode and source-based asymmetric IBFD (SAFD) transmission mode based on the different order of channel access, as shown in Fig. 1. All the nodes in the IBFD wireless networks have the same capability of IBFD communication and are given equal access priority.

As shown in Fig. 1, we define the transmission from node A to node B as the primary transmission. In this transmission, node A is primary transmitter (PT) and node B is primary receiver (PR). In IBFD dual transmission, we define the other transmission link as secondary 
transmission link, and its transmitter/receiver as secondary transmitter/receiver (ST and SR). In Fig. 1(b), the network carries out SFD transmission between node A and node B, where node A is both PT and SR, and node B is both PR and ST. In Fig. 1(c), DAFD transmission is conducted among nodes A, B, and D, where node A is PT, node B is both PR and ST, and node D is SR. In Fig. 1(d), nodes A, B, and D transmit packet in SAFD mode, where node A is both PR and SR, node B is PR, and node C is ST. We describe four transmission roles and any node in the IBFD wireless networks can be any one of the four roles.

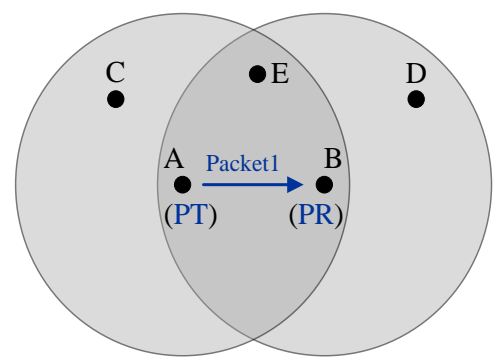

(a) HD

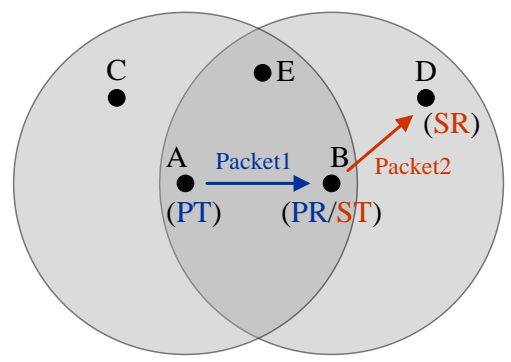

(c) DAFD

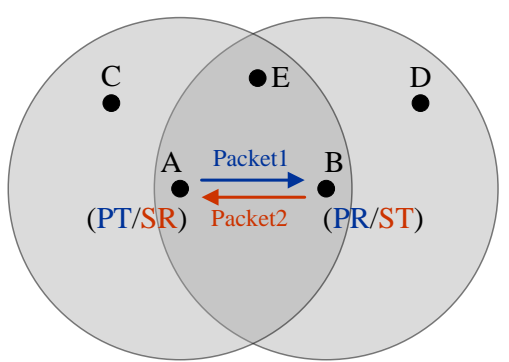

(b) SFD

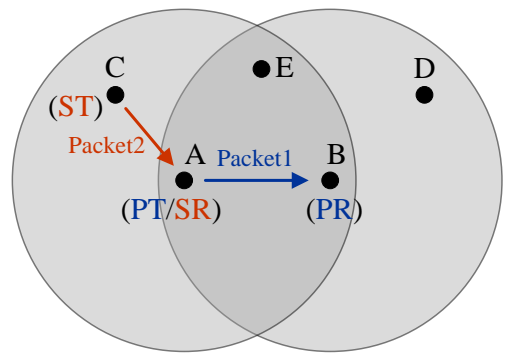

(d) SAFD

Fig. 1. The transmission modes in distributed IBFD wireless networks

Based on the assumption that self-interference has been suppressed below the noise floor [4] , we studied INI mitigation for asymmetric IBFD dual transmission. For the case of DAFD dual transmission link in Fig. 1(c), the Packet1 transmitted from node A would interfere with node $\mathrm{D}$, which intends to receive Packet2 from node $\mathrm{B}$. If node $\mathrm{D}$ is the exposed terminal of node A, and Packet1 transmitted from node A is sufficiently strong, then node D cannot receive the desired Packet2 correctly due to the INI caused by the Packet1 of node A. Therefore, it is essential to suppress INI for asymmetric dual transmission.

We define the received power at node $j$ from the packet transmitted by node $i$ as $P_{r, i-j}$, which is expressed as

$$
P_{r, i-j}=\alpha \cdot d_{i j}^{-n_{p}} P_{t, i},
$$

where $P_{t, i}$ is the transmit power of node $i, \alpha$ is a constant, $n_{p}$ is the path-loss exponent, $d_{i j}$ is the distance between node $i$ and node $j$, and $\alpha \cdot d_{i j}^{-n_{p}}$ is the deterministic path-loss [27]. Both $\alpha$ and $P_{t, i}$ are identical for all the transmitted frames. Thus, we can see that the received power $P_{r, i-j}$ is inversely proportional to the distance $d_{i j}$, which would be one of the criterions for a node to decide whether itself is suitable to contend the secondary link of SAFD dual transmission. For example, as shown in Fig. 1(d), node E can receive frames from both node $\mathrm{A}$ and $\mathrm{B}$, and then calculate out the distance $d_{A E}$ and $d_{B E}$. If a SAFD transmission occurs 
among node A, B and one neighbor of node A, node $\mathrm{E}$ will not be the proper neighbor node since it is close to node $B$ and interferes the normal reception of node $B$.

In Fig. 1(c), the SINRs of node B and node D receiver can be expressed as

$$
\begin{aligned}
& \operatorname{SINR}_{B}=\frac{P_{r, A-B}}{P_{S I}+n_{B}}, \\
& \operatorname{SINR}_{D}=\frac{P_{r, B-D}}{P_{r, A-D}+n_{D}},
\end{aligned}
$$

Where $n_{B}$ and $n_{D}$ are the white Gaussian noises at node $\mathrm{B}$ and $\mathrm{D}$, which is of zero mean and with unit variance; $P_{S I}$ is the power of residual self-interference signal known by node $\mathrm{B}$ itself after advanced self-interference cancellation techniques. For the successful reception of primary and secondary transmissions, the minimum of $\operatorname{SINR}_{B}$ and $\operatorname{SINR}_{D}$ must be higher than a SINR threshold $\beta$, i.e., $\operatorname{SINR}_{B} \geqslant \beta, \operatorname{SINR}_{D} \geqslant \beta$, where $\beta$ is calculated by the quality of service requirements of each node instead of the minimal SINR level that can support different modulations [8]. However, if node $\mathrm{A}$ is close to node $\mathrm{D}$, the value of $P_{r, A-D}$ might be too high to suffice the constraint condition of $\operatorname{SINR}_{D} \geqslant \beta$. Thus, node A and node B need to optimize their transmit powers respectively to maximize the value of $\operatorname{SINR}_{B}$ and $S I N R_{D}$ before data transmissions.

Communication nodes transmit control frames for medium access in HD mode, the component of received signals are purer than IBFD transmission. The PHY layer of the receiver is easier to obtain the value of received power. Thus, we plan to use control frame transmission to deliver some parameters for PHY layer to suppress INI. The primary transmitter node A and secondary transmitter node B both estimate $S I N R_{B}$ and $S I N R_{D}$ based on the received power $P_{r, A-B}, P_{r, B-D}$ and $P_{r, A-D}$. Next, they maximize the minimum of $\operatorname{SINR}_{B}$ and $\operatorname{SINR}_{D}$ while satisfying the SINR constraint condition. Based on the optimal SINR, node A and node B can calculate the optimal transmit power required for data transmission. After adjustment of transmit power, the received power of node D from node $\mathrm{A}$ can decrease so that it does not affect the ability of node $\mathrm{D}$ to receive the desired packet.

To realize this method, the key problem that we must solve firstly is how to improve the ability of node A and node B to obtain the information of received power $P_{r, A-B}, P_{r, B-D}$ and $P_{r, A-D}$. In this work, we add the received power information in the control frame. Then, by our proposed FD-DMAC three-way handshaking procedure, nodes A and B can both obtain the information required for SINR estimation. The specific medium access control mechanism and received power delivery will be described in Section 4.

\section{FD-DMAC mechanism}

In asymmetric IBFD dual communication, standard RTS/CTS mechanism can only establish the primary transmission link, but cannot reserve the secondary transmission channel. Thus, the distributed IBFD wireless networks with RTS/CTS scheme require two channel accesses and data transmissions to complete asymmetric dual communication, which will work equivalently to HD wireless networks. In this section, we describe the proposed FD-DMAC mechanism that is able to establish a symmetric or an asymmetric IBFD dual link by three-way handshaking. 


\subsection{Control frame structures}

We design the control frame structures of FD-DMAC mechanism based on those of standard 802.11 RTS/CTS frame. They contain three request frames RTS1, RTS2, and RTS3, and one response frame DCTS (Duplex CTS), as shown in Fig. 2. SA, DA, TM, and Pr represent the source address, destination address, transmission mode, and received power respectively. The frame control, packet duration and FCS (Frame Check Sequence) keep consistent with standard 802.11 [28]. Here the Frame Control field consists of protocol version, type, subtype and so on. The packet duration field contains the time required to transmit the packet of destination node. The FCS field is a 32-bit field containing a 32-bit CRC.

There are four IBFD transmission modes in distributed wireless networks. We only use 2 bits (which is marked as 2/8 bytes in Fig. 2) to denote TM field in control frame, i.e., '00' for HD mode, '01' for SFD mode, '10' for DAFD mode, and '11' for SAFD mode. After a communication node receiving control frame successfully, its physical layer uploads the value of received power to MAC layer and then the MAC layer store the value in the Pr field. FD-DMAC is a three-way handshaking medium access mechanism and the received power information will be obtained after the first handshaking. Thus, only the latter two handshaking can delivery received powers by control frame. Without loss of generality, we consider the value of received power is between -80 and $0 \mathrm{dBm}$, which can be represented in 8 bits. The first bit is used to indicate the sign of the value and the last 7 bits give an absolute value of received power. Thus, we design the Pr field to store two values of received power which are used to estimate SINR, and the field only occupies 2 bytes totally.

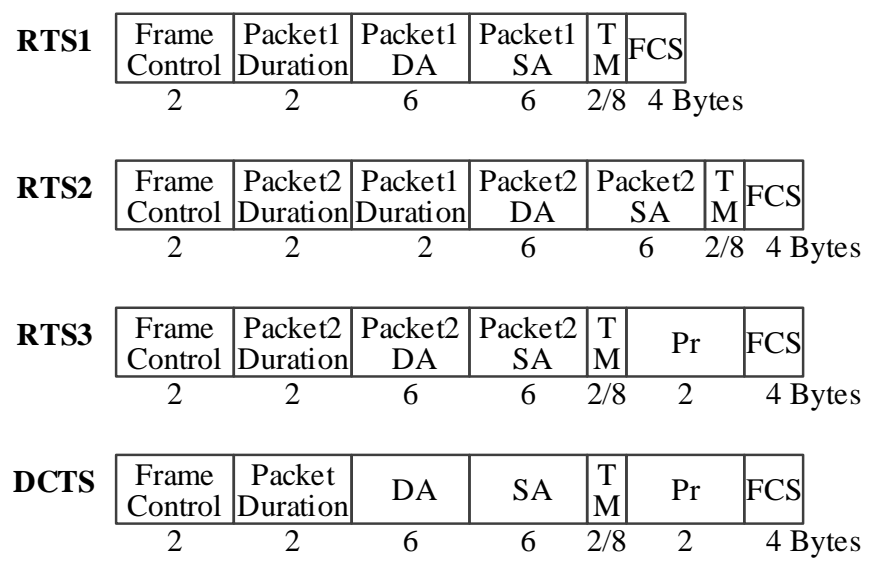

Fig. 2. Control frame structures

As shown in Fig. 2, RTS1 is used for node A to access the channel and request SFD transmission and its TM is ' 01 '. Node B requests DAFD transmission by sending RTS2 frame with '10'. Using RTS2, node B notifies its neighbors (containing node A) that it will receive Packet1 from node A and transmit Packet2 to node D simultaneously. Node C utilizes RTS3 with '11' to allow SAFD transmission. At the same time, it sends the received power information that is stored in RTS3 to node A. Node B and node D use DCTS to response to the channel access request, where the TM is set according to the transmission status of node B. The information in the Pr field of DCTS will assist the solicited nodes of node B and node D to adjust their transmit powers. Additionally, we set RTS2, RTS3 and DCTS to the same frame length to facilitate calculation of the duration of channel access. 


\subsection{FD-DMAC description}

We assume that each node in the distributed IBFD wireless network is able to sense channel status and its neighbors' status, irrespective of its transmitting status. If a node finds that the channel is idle for the duration of DIFS, it starts the backoff procedure and initiates a transmission while the backoff time counts down to 0 . If the node senses a busy channel, it must freeze its backoff counter and wait.

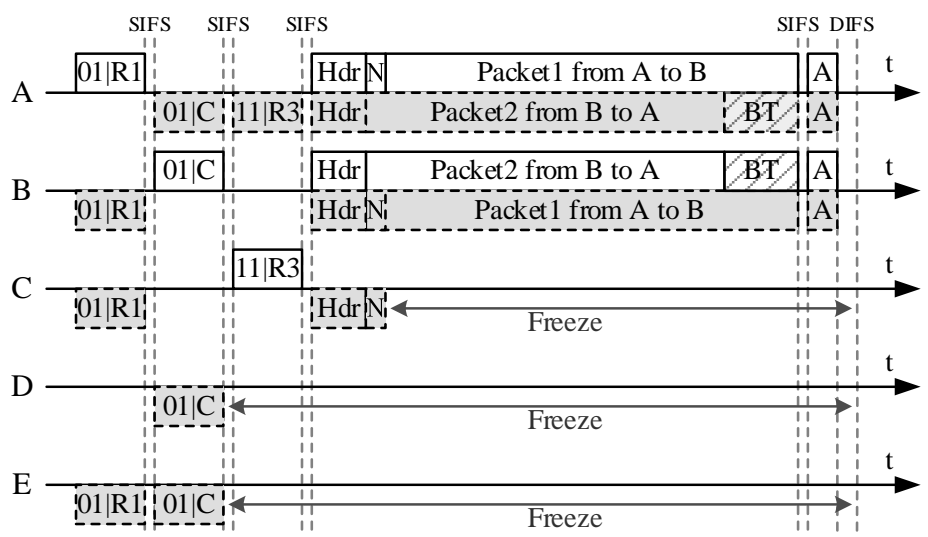

(a) Successful symmetric IBFD dual link transmission

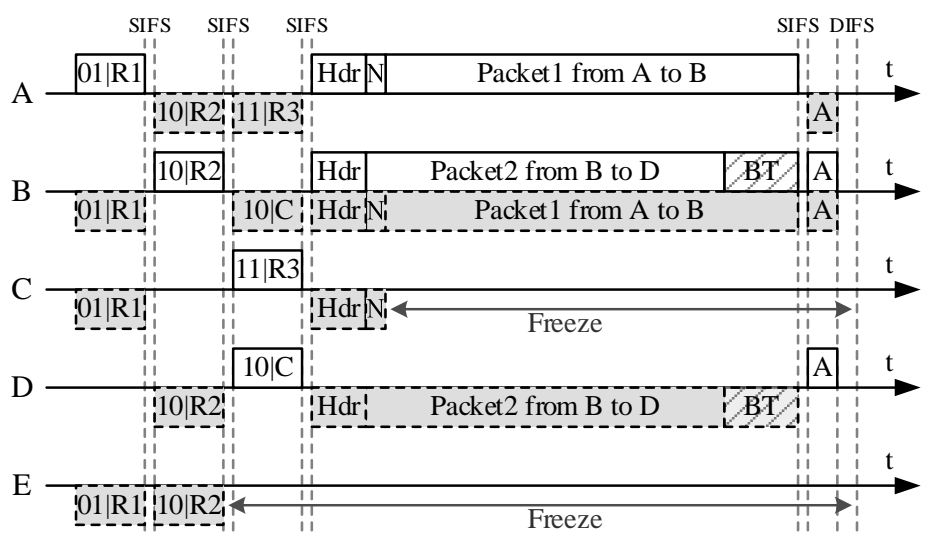

(b) Successful destination-based asymmetric IBFD dual transmission

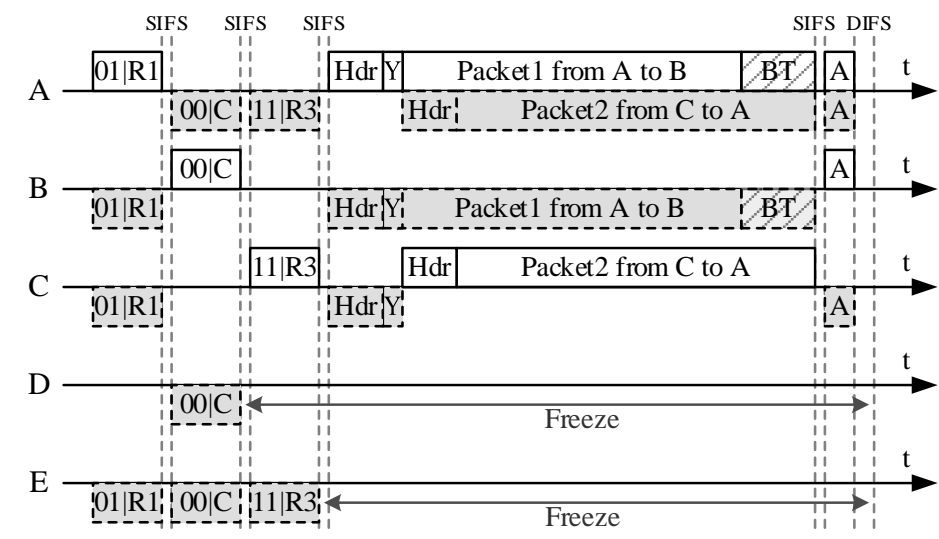

(c) Successful source-based asymmetric IBFD dual transmission 


\begin{tabular}{|c|c|c|c|c|c|}
\hline $01 \mid \mathrm{R} 1$ & RTS1 TM: 01 & $10 \mid \mathrm{R} 2$ & RTS2 TM: 10 & $11 \mid \mathrm{R} 3$ & RTS3 TM: 11 \\
\hline $00 \mid \mathrm{C}$ & DCTS TM: 00 & $01 \mid \mathrm{C}$ & DCTS TM: 01 & \begin{tabular}{|l|}
$10 \mid C$ \\
\end{tabular} & DCTS TM: 10 \\
\hline $\mathrm{Hdr}$ & Packet header & $\mathrm{BH} / \mathrm{Z}$ & Busytone & $\mathrm{A}$ & ACK \\
\hline
\end{tabular}

Fig. 3. Successful IBFD dual transmission

In order to better illustrate the FD-DMAC mechanism, we test three cases of IBFD wireless transmission of symmetric dual transmission, destination-based asymmetric dual transmission, and source-based asymmetric dual transmission, as shown in Fig. 3. The white portion upon the time axis indicates the control packet and data packet transmission and the gray part below the time axis indicates control packet and data packet reception. In addition to the communication nodes, we also analyze the behavior of neighbor nodes. The specific medium access procedure and data transmission are detailed as follows.

\section{1) Symmetric IBFD dual transmission}

Medium access: Fig. 3(a) shows the symmetric IBFD dual transmission. Node A wins the contention and then transmits RTS1 with 01 to node B for SFD transmission. After receiving RTS1 correctly and knowing the request of node A, if node B also has a packet (Packet2) to transmit to node A, it waits for a SIFS time, and then transmits DCTS with 01 back to node A. Node C, one of the nodes who have packets to node A, is exposed to node A but hidden to node $B$, and then would only receive RTS1 from node A but cannot receive DCTS from node $B$. Thus, node $C$ does not make sure that whether node $B$ has a packet to transmit. To contend the uplink transmission opportunity of node $A$, node $C$ expects to transmit a SAFD transmission request to node A. So in FD-DMAC mechanism, we set that node A and node B will not start IBFD data transmission immediately after node A finishes reception from node B but instead reserve the time for node C to send RTS3. After receiving RTS1 from node A, node $C$ waits for the duration of SIFS+DCTS+SIFS, and then transmits RTS3 with 11 for SAFD transmission. Node A will respond to the request of node $C$ during data transmission procedure. Nodes $\mathrm{D}$ and $\mathrm{E}$, the exposed terminals of node $\mathrm{B}$, can obtain the information of SFD transmission between nodes A and B. Then they freeze their backoff counters and wait based on the longer length of Packet1 and Packet2. After this three-way handshaking, the symmetric IBFD dual link between node A and node B is established.

Data transmission: After node A receives DCTS from node B successfully, both node A and node B wait for a SIFS + RTS3 + SIFS time, and then transmit their respective packets to each other simultaneously in SFD mode. The time of the packet transmission lasts for the longer duration of Packet1 and Packet2. As shown in Fig. 3(a), the length of Packet1 is longer than that of Packet2, which is known by node B. Therefore, even if node B finishes transmitting Packet2, it will send busytone to keep occupying the channel and wait until Packet1 transmission is finished before sending ACK to node A. Moreover, we add a flag between the packet header and data to reply to the SAFD transmission request of node C. If node A agrees with this request, it sets the flag to 1 (designated as $\mathrm{Y}$ in Fig. 3). If not, the flag is set to 0 (designated as $\mathrm{N}$ in Fig. 3). The flag only occupies 1 bit. In SFD transmission, node A disagrees obviously the request of node $\mathrm{C}$ and sets the flag as 0 . Furthermore, when one more nodes like node $\mathrm{C}$ who just have packets to node $\mathrm{A}$ contend uplink transmission simultaneously with node $\mathrm{C}$, node A will not receive the requests clearly and then set the flag 
to 0 to refuse all the nodes. As soon as node $\mathrm{C}$ receives the header and flag of Packet 1 from node $\mathrm{A}$, node $\mathrm{C}$ realizes that its request is refused, and then freezes its backoff counter and waits. After data dual transmission, both node A and node B send an ACK to each other simultaneously after a SIFS time to finish the symmetric IBFD dual transmission.

\section{2) Destination-based asymmetric IBFD dual transmission}

Medium access:Fig. 3(b) shows the destination-based asymmetric IBFD dual transmission, where node A contends the channel and initiates a RTS1 with 01 to node B for SFD transmission. Node B receives and reads RTS1. However, node B has no packet to transmit to node A but expects to transmit a packet (Packet2) to its neighbor node D. Thus after a SIFS time, node B transmits a RTS2 with 10 to node D for DAFD transmission. Here RTS2 is a request frame and also acts as a response role. Node A receives RTS2 and is notified that node B will receive Packet1 from node A and transmit Packet2 to node D simultaneously, i.e. node $\mathrm{B}$ will communicate in DAFD mode. Node D agrees with the request of node B, and then returns a DCTS with 10 to node B after a SIFS time. The behaviors of nodes C and $\mathrm{E}$ in DAFD transmission are similar to that in SFD transmission.

Data transmission: After node A receives RTS3 from node C and node B receives DCTS from node D successfully, node A transmits Packet1 to node B and node B transmits Packet2 to node D simultaneously in DAFD mode. The time of the packet transmission lasts for the longer duration of Packet1 and Packet2. Node B, the node who first finishes packet transmission, sends busytone to occupy the channel and notify the transmission status to its neighbor. Node A disagrees with the SAFD transmission request of node C. Thus, it sets the flag behind the Packet1 header to 0. After receiving the header and flag of Packet1 from node $\mathrm{A}$, node $\mathrm{C}$ freezes its backoff counter and waits. After data dual transmission among nodes A, B, and D, node B sends an ACK to node A and node D sends an ACK to node B simultaneously after a SIFS time to complete the DAFD dual transmission.

\section{3) Source-based asymmetric IBFD dual transmission}

Medium access: Fig. 3(c) shows the source-based asymmetric IBFD dual transmission, in which node A achieves the channel and sends a RTS1 with 01 to node B for SFD transmission. However, node B has no packet to transmit to node A or its neighbors. Thus after receiving RTS1, node B waits for a SIFS time and transmits a DCTS with 00 to notify node A of its future transmission plan. The ' 00 ' represents that node B will only receive packet during packet transmission but does not mean that node A will not transmit in IBFD mode. The neighbor nodes, who can receive the ' 00 ', may contend the uplink transmission opportunity of node A to keep the dual packet transmission in the network. The behavior of node $C$ in SAFD transmission is similar to that in SFD transmission. Node D, the exposed node of node $\mathrm{B}$ and the hidden node of node $\mathrm{A}$, can receive DCTS from node B and knows that node $\mathrm{B}$ will be busy receiving. Thus node $\mathrm{D}$ freezes its backoff counter to avoid the interference to the reception of node B. Node E, the expose node of both node A and B, is able to receive RTS1 from node A and DCTS from node B and then know that the uplink of node A might be free during data transmission. After calculate out the distance between node B and node $\mathrm{E}$ (i.e. $d_{B E}$ ) by $P_{r, B-E}$ in (1), node $\mathrm{E}$ realize that itself is close to node B. Thus, even if node $\mathrm{E}$ has a packet to node $\mathrm{A}$, it will not contend the secondary link of the SAFD transmission to increase the interference to node B. As a result, node E freezes its backoff counter and waits. 
Data transmission: After receiving RTS3 from node C successfully, node A waits for a SIFS time and then transmits Packet1 to node B. Node A agrees with the SAFD transmission request from node $\mathrm{C}$. Therefore, it sets the flag behind the Packet1 header to 1. After receiving the header and flag of Packet1 from node A, node $\mathrm{C}$ realizes that node A has accepted its request and then transmits Packet2 to node A immediately. Node A transmits Packet1 to node B and receives Packet2 from node C in SAFD mode. The time of the packet transmission depends on the longer duration of Packet1 and Header + Packet2. Since node A knows the packet lengths of both itself and node $\mathrm{C}$, it continues to occupy the channel by sending busytone if it first finishes packet transmission. When node $\mathrm{B}$ no longer receives signal from node $A$, it can tell that both node $A$ and $C$ have completed dual packets transmission. After data dual transmission among nodes A, B, and C, node B sends an ACK to node A and node A sends an ACK to node C simultaneously after a SIFS time to end the SAFD dual transmission.

\subsection{Received power delivery}

We set all the nodes in the IBFD wireless networks to have the same transmit powers during three-way handshaking. Since communication nodes transmit control frame in HD mode, the component of received signals are purer than IBFD transmission. Additionally, communication nodes only estimate the value of SINR for judgement. Then, it can be considered that the received power of node $j$ from node $i$ is equal to the received power of node $i$ from node $j$, i.e., $P_{r, i-j}=P_{r, j-i}$. As shown in Section 3, if the communication nodes are exposed to each other, primary transmitter node $\mathrm{A}$ and secondary transmitter node $\mathrm{B}$ require the value of $P_{r, A-B}, P_{r, B-D}$, and $P_{r, A-D}$ to estimate $S_{I N R_{B}}$ and $S I N R_{D}$ in DAFD dual transmission, and primary transmitter node $A$ and secondary transmitter node $C$ require the value of $P_{r, C-A}$, $P_{r, A-B}$, and $P_{r, C-B}$ to estimate $S I N R_{A}$ and $S I N R_{B}$ in SAFD dual transmission. By receiving control frames, a node can obtain its received powers and remember them. It then adds the received power values in the Pr field of its control frame which will be sent to the destination node. As shown in Fig. 4, after FD-DMAC medium access, the primary transmitter and secondary transmitter can achieve their required received power values (which are indicated in red) to estimate SINR after three-way handshaking medium access.

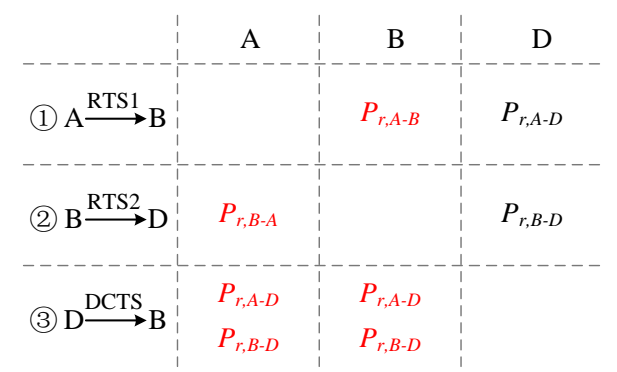

(a)

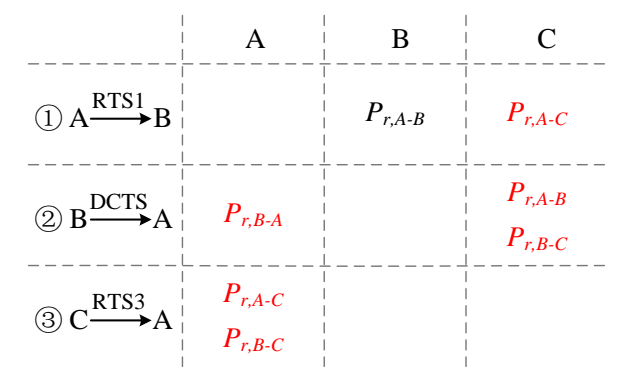

(b)

Fig. 4. Received power deliveries in (a) DAFD dual link; (b) SAFD dual link

\section{Theoretical analysis}

Taking advantage of the similarity between our proposed FD-DMAC mechanism and RTS/CTS mechanism, we use the discrete-time Markov model in [28] to analyze the performance of distributed IBFD wireless networks with the FD-DMAC mechanism. We 
study a distributed wireless network that consists of $n$ nodes. All the nodes in the network can listen to the channel, capture IBFD wireless transmission opportunities, and detect collisions.

Before sending a packet on the channel, each node should set a random back-off number from the contention window based on transmission history. The window is defined as $\left(0, W_{i}\right)$, where $W_{i}=2^{i} W, i=0,1,2, \ldots, m, W$ is the minimum contention window and $m$ is the maximum backoff stage. From this, the contention window can expand up to the maximum value of $2^{m} W$. Based on the Markov model of the backoff window size, the probability of a node transmitting in a random chosen slot time, denoted as $\tau$, can be calculated as [28]:

$$
\tau=\frac{2}{1+W+p W \sum_{i=0}^{m-1}(2 p)^{i}},
$$

where $p$ is the conditional collision probability. If a node transmits a packet, a collision happens when at least one of the other $n-1$ nodes is currently transmitting. So $p$ is given as:

$$
p=1-(1-\tau)^{n-1} .
$$

We define the probability that there exists at least one transmission during the slot time as $P_{t r}$, which can be expressed as:

$$
P_{t r}=1-(1-\tau)^{n} .
$$

Then, the probability of idle time during the slot time can be given as 1- $P_{t r}$. The channel idle time in the slot time is $\left(1-P_{t r}\right) \sigma$, where $\sigma$ is the duration of the idle time.

\subsection{Throughput}

Let $S$ be the normalized system throughput, defined as the fraction of the total time used to send payload successfully during a given slot time. To compute $S$, we need to first calculate the probabilities of successful and failed IBFD transmissions.

In the IBFD wireless network, if node B has a packet to transmit to its neighbor, the SAFD transmission will not happen. Here we define the probability of secondary transmitter node B having packets to transmit to its neighbors as $\lambda$. When node B transmits its packet to node A or node D, i.e., the communication nodes transmit the packets in SFD or DAFD mode, the successful transmission probability, denoted as $P_{s 1}$, is given as:

$$
P_{s 1}=\left(\begin{array}{l}
n \\
1
\end{array}\right) \tau(1-\tau)^{n-1} \lambda
$$

and the transmission time lasts $T_{s 1}=R T S 1+D C T S$ (or RTS2) $+R T S 3+H+N+E[P]_{\text {longer } 1}+$ $A C K+4 *$ SIFS + DIFS. Here $H=P H Y_{h d r}+M A C_{h d r}$ is the transmission duration of packet header, $E[P]_{\text {longer } 1}$ is the longer payload size of Packet1 and Packet2. Since the flag behind the packet header only occupies 1 bit, we ignore the flag bit when we compute the length of $E[P]_{\text {longer } 1 \text {. }}$

When node B has no packet to transmit and node C can capture the opportunity of SAFD transmission, the successful transmission probability, denoted as $P_{\mathrm{s} 2}$, is given as:

$$
P_{s 2}=\left(\begin{array}{l}
n \\
1
\end{array}\right) \tau(1-\tau)^{n-1}(1-\lambda),
$$

and the transmission time lasts $T_{s 2}=R T S 1+D C T S+R T S 3+H+Y+E[P]_{\text {longer } 2}+A C K+$ $4 * S I F S+D I F S$. Here $E[P]_{\text {longer } 2}$ is the longer payload size of Packet1 and Header + Packet2. 
Since RTS2, RTS3, and DCTS have the same frame length, the main difference between $T_{s 1}$ and $T_{\mathrm{s} 2}$ are the different transmission durations of $E[P]_{\text {longer } 1}$ and $E[P]_{\text {longer } 2 \text {. }}$

The failed IBFD transmission in the slot time occurs when more than one of the nodes transmit RTS1 simultaneously. This probability, denoted as $P_{c}$, can be given as:

$$
P_{c}=1-(1-\tau)^{n}-\left(\begin{array}{l}
n \\
1
\end{array}\right) \tau(1-\tau)^{n-1},
$$

and the collision time lasts $T_{c}=R T S 1+D I F S$.

Now, the throughput $S$ can be calculated as:

$$
S=\frac{\left(P_{s 1}+P_{s 2}\right) E[P]}{\left(1-P_{t r}\right) \sigma+P_{s 1} T_{s 1}+P_{s 2} T_{s 2}+P_{c} T_{c}},
$$

where $E[P]$ is the total packet payload size of the two simultaneous transmitted packets, i.e., the amount of payload information successfully transmitted within a slot time.

\subsection{Delay}

When transmitting two packets, the IBFD wireless network saves nearly half of the time as compared to the HD wireless network, since the simultaneously dual packets transmission after channel access. Here we define the delay as the duration from a packet of primary transmitter coming to the front of the queue to its successful reception as well as the packet of secondary transmitter to its reception. Let $D$ be the delay of the IBFD wireless network with FD-DMAC mechanism. The average value of $D$, denoted as $E[D]$, can be given as [29]:

$$
E[D]=N_{c}\left(T_{b d}+T_{c}+T_{o}\right)+\left(T_{b d}+T_{s}\right) .
$$

Here, $N_{c}$ is the number of collisions of a frame until its successful reception, $T_{b d}$ is the average backoff delay that a node uses before accessing the channel again. $T_{o}$ is the time that a node has to wait before sensing the channel again when the frames collide during transmission, which can be given as $T_{o}=$ SIFS + CTS_timeout. $T_{s}$ is the successful transmission time, which is expressed as $T_{s}=\lambda T_{s 1}+(1-\lambda) T_{s 2}$. Finally, $T_{c}$ was given above.

The average number of retransmissions is given as $1 / P_{s}$, and the last transmission must be successful. Then the number of collisions $N_{c}$ can be calculated as:

$$
N_{c}=\frac{1}{P_{s}}-1
$$

The average backoff delay $T_{b d}$ is determined based on the initial value of the backoff counter and the duration of the time during which the counter continues freezing. The initial value of the counter is defined as a random variable $X$, and its average value as $E[X]$, which can be calculated based on the Markov model without considering the counter stop:

$$
E[X]=\frac{\tau(1-p)}{6}\left[\sum_{i=0}^{m-1} p^{i}\left(W_{i}^{2}-1\right)+\frac{p^{m}}{1-p}\left(W_{m}^{2}-1\right)\right] .
$$

The backoff counter freezes when the channel stays in busy or collision status. We denote the time that the counter freezes as $F$, and the average time of $F$ as $E[F]$, which can be given as:

$$
E[F]=E\left[N_{F}\right]\left(P_{s 1} T_{s 1}+P_{s 2} T_{s 2}+P_{c} T_{c}\right),
$$


where $E\left[N_{F}\right]$ is the average number of times that a node freezes before its counter reaches 0 . The counter keeps continuously decreasing before it freezes. Thus the mean number of times that the counter works consecutively can be expressed as $\left(1-P_{t r}\right) \sigma$. Then, $E\left[N_{F}\right]$ is expressed as:

$$
E\left[N_{F}\right]=\frac{E[X]}{\max \left(\left(1-P_{t r}\right) \sigma, 1\right)}-1
$$

Based on (13) and (14), we can calculate the average backoff delay as $T_{b d}=E[X]+E[F]$. Finally, combining the above equations, the average frame delay $E[D]$ in (11) can be determined.

\section{Numerical results}

We use MATLAB to evaluate the performance of the FD-DMAC mechanism for distributed IBFD wireless networks and validate our analytical model. The basic wireless communication parameters for the simulation are listed in Table 1. To show the advantage of our mechanism, we separately compared the performance of FD-DMAC mechanism to that of the RTS/CTS [28] mechanism for HD wireless networks and RTS/FCTS [10] mechanism proposed in for IBFD wireless networks.

Table 1. Parameters for numerical results

\begin{tabular}{cccc}
\hline Parameter & Value & Parameter & Value \\
\hline Packet1 payload & 8184 bits & FCTS[10] & 528 bits \\
Packet2 payload & 8184 bits & ACK & 240 bits \\
PHY header & 128 bits & flag & 1 bit \\
MAC header & 272 bits & Channel Bit Rate & $1 \mathrm{Mbit} / \mathrm{s}$ \\
RTS1 & 290 bits & Slot Time $(\sigma)$ & $50 \mu \mathrm{s}$ \\
RTS2/RTS3/DCTS & 306 bits & SIFS & $28 \mu \mathrm{s}$ \\
RTS & 288 bits & DIFS & $128 \mu \mathrm{s}$ \\
CTS & 240 bits & CTS_timeout & $300 \mu \mathrm{s}$ \\
\hline
\end{tabular}

Fig. 5 shows that the system throughput depends on the number of nodes in the distributed wireless network. Here the maximum backoff stage $m$ is set to 6 , and the probability of node $\mathrm{B}$ having packets to transmit $\lambda$ is set to 0.8 . We can see that by FD-DMAC mechanism, the throughput of the distributed IBFD network is significantly improved. For the case of minimum contention window $W=16$, the throughput of IBFD network with FD-DMAC is about 1.59, and that of HD network with RTS/CTS is about 0.83. As a result, it is clear to illustrate the advantage of IBFD wireless communication that it improves throughput by about $90 \%$ as compared to HD. And compared with the IBFD network with RTS/FCTS mechanism which has longer channel access time, the IBFD network with FD-DMAC mechanism achieved over $6.97 \%$ maximum throughput gains. In previous research [28, 29], we know that the basic access which does not need medium access request has a strong throughput decrease with the expansion of network scale. Thus, thanks to the MAC mechanisms with access request before packet transmission, the nodes can decide whether to transmit packets or not with small cost, which bring gently throughput decrease. 


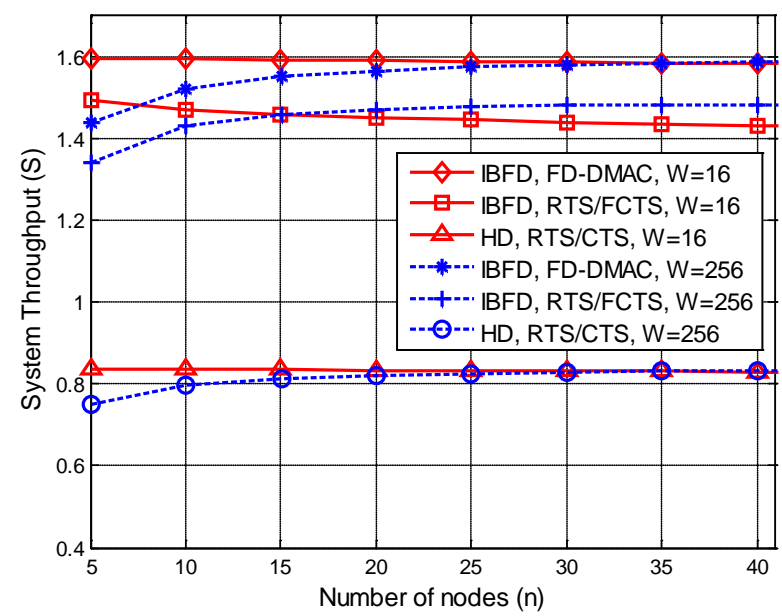

Fig. 5. The system throughput versus the number of stations for different access mechanisms and minimum contention window sizes

We also analyze the throughput of the IBFD network with FD-DMAC with respect to the minimum contention window, as shown in Fig. 5. We find that for $W=16$, as the number of nodes increases, the throughput decreases slowly. For $W=256$ that the backoff time will be longer, the throughput continues to increase. In other words, if the nodes are configured with a longer backoff time, they will experience more time before channel competition and hence avoid request collisions, keeping a higher system throughput in a large scale network. It is thus clear that the network needs to select a proper contention window size based on the number of nodes to maximize throughput. Under the same condition of minimum contention window size, the throughput of IBFD mechanism with FD-DMAC varies more slowly as the number of nodes increases. Thus, our proposed mechanism can be utilized in large networks and provides higher performance than the MAC scheme for the three-node network model proposed in [16].

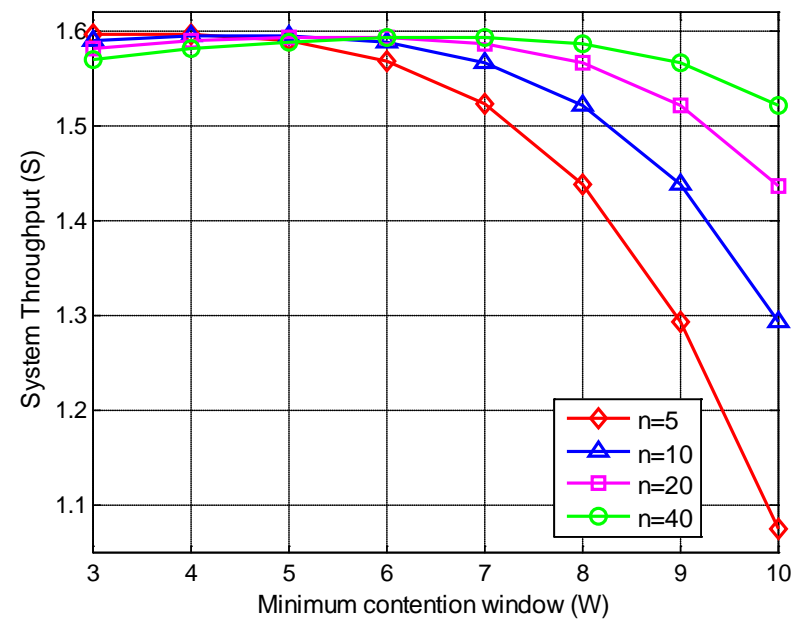

Fig. 6. The system throughput versus the minimum contention window for FD-DMAC mechanism. 


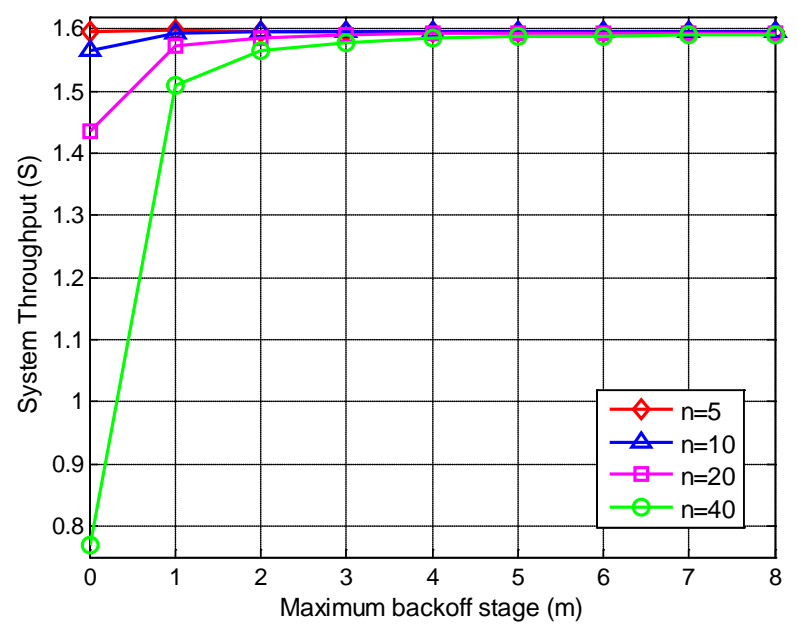

Fig. 7. The system throughput versus the maximum backoff stage for FD-DMAC mechanism.

The initial value of the backoff counter is related to minimum contention window and maximum backoff stage. To choose an appropriate initial value of the counter, we analyze the system throughput versus the minimum contention window size and the maximum backoff stage for IBFD network with FD-DMAC, as shown in Fig. 6 and Fig. 7, respectively. In Fig. 6, the maximum backoff stage $m$ is fixed to 6. We determined the relationship between minimum contention window and throughput to select a proper $W$ for different network scale. For 10 nodes, the maximum throughput is achieved at a $W$ of 16 . For 20 nodes, the network selects 64 as the minimum contention window size for maximum throughput. In Fig. 7, the minimum contention window $W$ is set to 16 . Then, we analyze the effect of maximum backoff stage $m$ on network throughput. Similarly, we can also choose a proper $m$ for different network scale. Overall, when the number of nodes increases, the network requires increasing $W$ and $m$ to enlarge the initial value of the backoff counter and avoid collision.

Fig. 8 compares the system throughput for different mechanisms and shows the throughput depends on the transmission probability $\tau$. Here, the probability of node B having packets to transmit $\lambda$ is set to 0.5. The maximum throughput of IBFD network with FD-DMAC is about 1.61, also indicating the double throughput compared with HD network with RTS/CTS. With the same network scale $n=20$, the IBFD network with FD-DMAC achieves over $11.29 \%$ maximum throughput gains over IBFD network with RTS/FCTS. As the increase of the transmission probability $\tau$, collision is more likely to occur, so that the throughput of the three mechanisms all decrease. However, the throughput of our mechanism is always higher than that of the other two. 


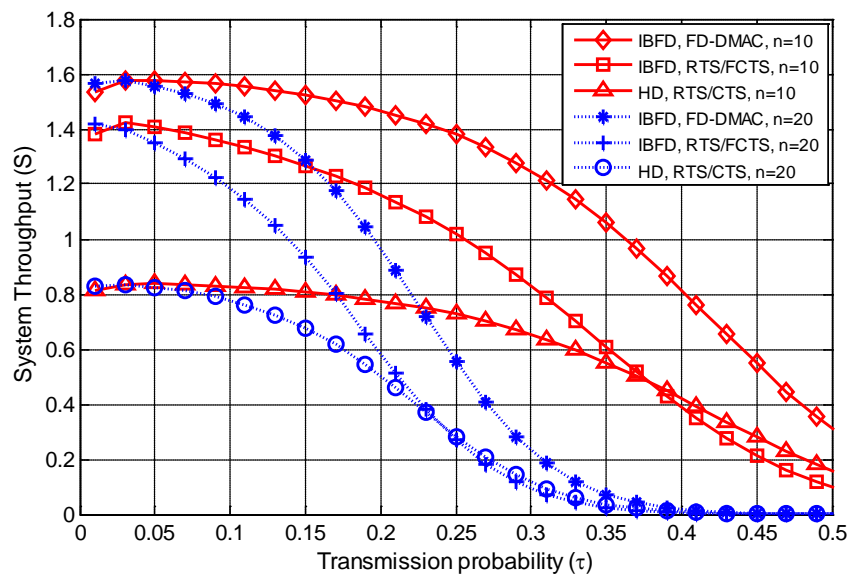

Fig. 8. The system throughput versus the transmission probability for different mechanisms and numbers of nodes.

Comprehensively analyzing Fig. 5 and Fig. 8, we can see that the probability of node B having packets to transmit $\lambda$ has little influence on the throughput of IBFD network with FD-DMAC, but has an obvious impact on that of IBFD network with RTS/FCTS. To verify this point, we analyzed the system throughput versus the transmission probability $\tau$ and the probability of node $\mathrm{B}$ having packets to transmit $\lambda$ for IBFD network with different mechanisms, as shown in Fig. 9. When the transmission probability is a fixed value, the throughput of IBFD network with FD-DMAC remains nearly invariable as $\lambda$ decreases, but the throughput of IBFD network with RTS/FCTS decreases continually. Thus, FD-DMAC mechanism remains more stable throughput for the IBFD wireless network.

To better validate the advantage of low delay of FD-DMAC mechanism, we also study the delay of two packets transmission versus the number of nodes for different access mechanisms, as shown in Fig. 10 and Fig. 11. As the number of nodes increases, the delay of the IBFD and HD networks both increases gradually. However, the delay of the IBFD network with FD-DMAC shows the slowest increase, and is always the lowest. Our results indicate that the FD-DMAC mechanism can better expand to a large scale network compared with RTS/CTS and RTS/FCTS mechanism.

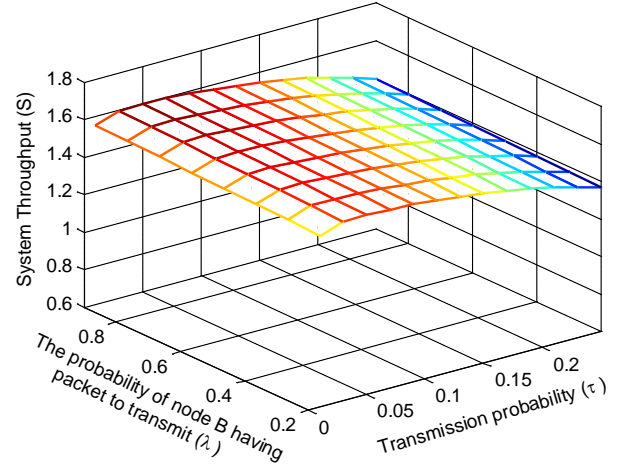

(a)

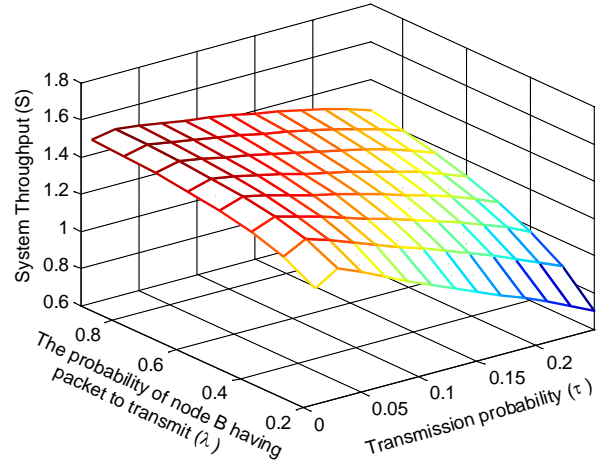

(b)

Fig. 9. The system throughput versus the transmission probability and the probability of node B having packets to transmit for IBFD wireless network with (a) FD-DMAC mechanism, (b) RTS/FCTS mechanism. 


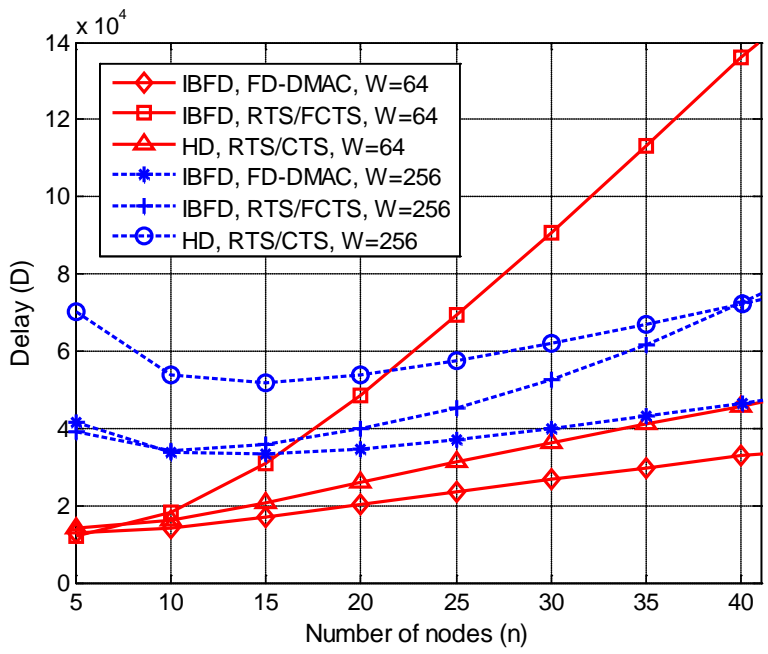

Fig. 10. The delay versus the number of nodes for different mechanisms and minimum contention window sizes.

As shown in Fig. 10, the maximum backoff stage $m$ is set to 3, the probability of node B having packets to transmit $\lambda$ is set to 0.6 , and the minimum contention window $W$ is set to 64 or 256 to analyze the relationship between minimum contention window and delay. For a fixed number of nodes, the delay increases as $W$ increases. As shown in Fig. 11, $W$ is set to $128, \lambda$ is maintained at 0.6 , and $m$ was set to 3 or 6 . We can see that the delay increases as $m$ increases in the same network scale. Comprehensively, when the initial value of backoff counter depended on a larger maximum backoff stage $m$ and minimum contention window $W$, the IBFD network with FD-DMAC suffers longer delays. This is the expense of higher throughput.

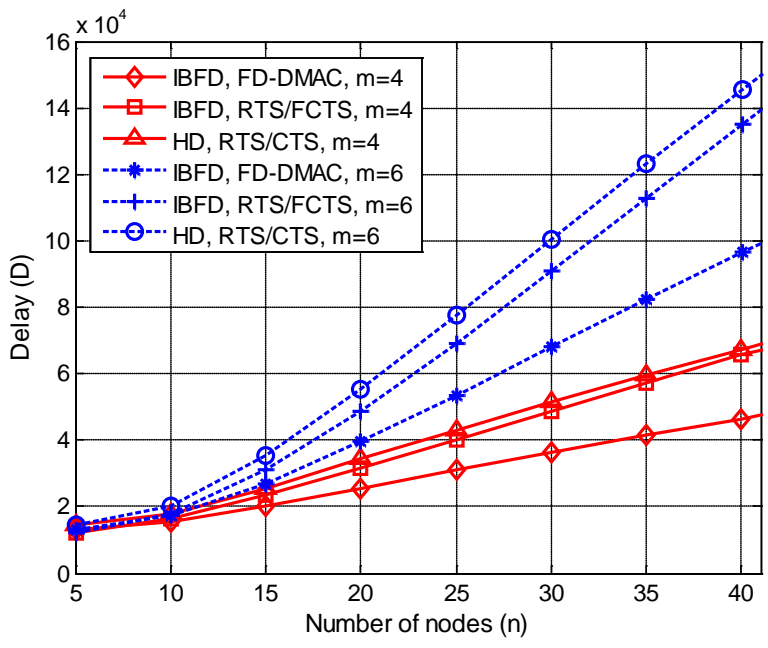

Fig. 11. The delay versus the number of nodes for different mechanisms and maximum backoff stages. 


\section{Conclusion}

We proposed a FD-DMAC mechanism for distributed IBFD wireless communication to provide greater asymmetric dual transmission opportunities with low INI. Compared with RTS/CTS, our proposed mechanism only requires single channel access to establish symmetric/asymmetric dual link for two-way simultaneous packet transmission. We defined control frames structures to implement our mechanism and assist communication nodes to adjust transmit power for INI suppression. After obtaining the information of channel transmission status by FD-DMAC medium access, the neighbors of communication nodes attempt asymmetric dual communication for further performance gain. Using a Markov model, we provided theoretical analysis and evaluated achievable throughput and delay. Results show that IBFD communication with FD-DMAC improves the throughput by $90 \%$ as compared to HD communication with RTS/CTS. Moreover, the transmission status of secondary transmitter has little influence on the throughput of FD-DMAC compared with RTS/FCTS in IBFD wireless networks.

\section{Acknowledgment}

This work is supported by the Fundamental Research Funds for China University of Mining and Technology (2014ZDPY16).

\section{References}

[1] S. Goyal, P. Liu, S. S. Panwar, and R. A. Difazio, "Full duplex cellular systems: will doubling interference prevent doubling capacity?” IEEE Communications Magazine, vol. 53, no. 5, pp. 121-127, 2015. Article (CrossRef Link)

[2] A. Sabharwal, P. Schniter, D. Guo, D. Bliss, S. Rangarajan and R. Wichman, "In-band full-duplex wireless: challenges and opportunities," IEEE Journal on Selected Areas in Communications, pp. 1637-1652, 2014. Article (CrossRef Link)

[3] D. Kim, H. Lee and D. Hong, "A survey of in-band full-duplex transmission from the perspective of PHY and MAC layers,” IEEE Communications Surveys \& Tutorials, pp. 1-31, 2015. Article (CrossRef Link)

[4] D. Bharadia, E. Mcmilin and S. Katti, "Full duplex radios," in Proc. of 2013 ACM Special Interest Group on Data Communication, vol. 43, no. 4, pp. 375-386, August 12-16, 2013. Article (CrossRef Link)

[5] A. Balatsoukas-Stimming, P. Belanovic, K. Alexandris and A. Burg, "On self-interference suppression methods for low-complexity full-duplex MIMO,” in Proc. of 2013 Asilomar Conference on Signals, Systems and Computers, pp. 992-997, November 3-6, 2013. Article (CrossRef Link)

[6] S. Goyal, P. Liu, O. Gurbuz, E. Erkip and S. Panwar, “A distributed MAC protocol for full duplex radio,” in Proc. Asilomar Conference on Signals, Systems and Computers, pp. 788-792, 2013. Article (CrossRef Link)

[7] A. Tang and X. Wang, “A-Duplex: Medium Access Control for Efficient Coexistence Between Full-Duplex and Half-Duplex Communications," IEEE Transactions on Wireless Communications, vol. 14, no. 10, pp. 5871-5885, 2015. Article (CrossRef Link)

[8] W. Choi, H. Lim, and A. Sabharwal, "Power-controlled medium access control protocol for full-duplex WiFi networks,” IEEE Transactions on Wireless Communications, vol. 14, no. 7, pp. 3601-3613, 2015. Article (CrossRef Link)

[9] M. Feng, S. Mao, and T. Jiang, "Duplex mode selection and channel allocation for full-duplex cognitive femtocell networks," in Proc. IEEE Wireless Communications and Networking Conference, pp. 1900-1905, 2015. Article (CrossRef Link) 
[10] W. Cheng, X. Zhang and H. Zhang, "RTS/FCTS mechanism based full-duplex MAC protocol for wireless networks,” in Proc. of 2013 IEEE Global Communications Conference, pp. 5017-5022, December 9-13, 2013. Article (CrossRef Link)

[11] X. Wang, A. Tang and P. Huang, "Full duplex random access for multi-user OFDMA communication systems,” Ad Hoc Network, vol. 24, no. A, pp. 200-213, 2015. Article (CrossRef Link)

[12] "Wireless Open Access Research Platform (WARP) Project." http://warpproject.org/. Rice University, Houston, TX, USA. Article (CrossRef Link)

[13] J. Y. Kim, O. Mashayekhi, H. Qu, M. Kazadiieva, and P. Levis, “Janus: A novel MAC protocol for full duplex radio,” CSTR, 2013. Article (CrossRef Link)

[14] “Universal Software Radio Peripheral (USRP).” http://www.ettus.com. Ettus Research LLC. Article (CrossRef Link)

[15] Z. Tong, C. Russ, S. Vanka, and M. Haenggi, "Prototype of virtual full duplex via rapid on-off-division duplex,” IEEE Transactions on Communications, vol. 63, no. 10, pp. 3829-3841, 2015. Article (CrossRef Link)

[16] K. Thilina, H. Tabassum, E. Hossain and D. Kim, "Medium access control design for full duplex wireless systems: challenges and approaches,” IEEE Communications Magazine, vol. 53, no. 5, pp. 112-120, 2015. Article (CrossRef Link)

[17] M. Jain, J. I. Choi, T. Kim, D. Bharadia, S. Seth, K. Srinivasan, P. Levis, S. Katti and P. Sinha, "Practical, real-time, full duplex wireless," in Proc. of 17th Annual International Conference on Mobile Computing and Networking, pp. 301-312, September 19-23, 2011. Article (CrossRef Link)

[18] S. Kim and W. E. Stark, “On the performance of full duplex wireless networks," in Proc. of 47th Annual Conference on Information Sciences and Systems, pp. 1-6, March 20-22, 2013. Article (CrossRef Link)

[19] R. Doost-Mohammady, Y. Naderi, and K. Chowdhury, "Performance analysis of CSMA/CA based medium access in full duplex wireless communications," IEEE Transactions on Mobile Computing, vol. 15, no. 6, pp. 1457-1470, 2016. Article (CrossRef Link)

[20] J. Seddar, H. Khalife, W. Safwi, and V. Conan, "A full duplex MAC protocol for wireless networks,” in Proc. IWCMC, pp. 244-249, 2015. Article (CrossRef Link)

[21] J. K. Kim, W. K. Kim, and J. H. Kim, "A new full duplex MAC protocol to solve the asymmetric transmission time,” in Proc. IEEE Globecom Workshops, pp. 1-5, 2015. Article (CrossRef Link)

[22] J. Bai and A. Sabharwal, "Distributed full-duplex via wireless side-channels: Bounds and protocols,” IEEE Transactions on Wireless Communications, vol. 12, no. 8, pp. 4162-4173, 2013. Article (CrossRef Link)

[23] S. Sen, R. Roy Choudhury, and S. Nelakuditi, "CSMA/CN: carrier sense multiple access with collision notification,” IEEE/ACM Transactions on Networking, vol. 20, no. 2, pp. 544-556, 2012. Article (CrossRef Link)

[24] T. Maksymyuk, M. Kyryk, and M. Jo, "Comprehensive spectrum management for heterogeneous networks in LTE-U,” IEEE Wireless Communications, vol. 23, no. 6, pp. 8-15, 2017. Article (CrossRef Link)

[25] H. Zuo, Y. Sun, C. Lin, S. Li, H. Xu, Z. Tan, and Y. Wang, “A three-way handshaking access mechanism for point to multipoint inband full-duplex wireless networks,” KSII Transactions on Internet and Information Systems, vol. 10, no. 7, pp. 3131-3149, 2016. Article (CrossRef Link)

[26] A. Sahai, G. Patel and A. Sabharwal, "Pushing the limits of full duplex: design and real-time implementation,” Rice University Technical Report TREE1104, 2011. Article (CrossRef Link)

[27] Z. Hadzi-Velkov and B. Spasenovski, "Capture effect in IEEE 802.11 basic service area under influence of Rayleigh fading and near/far effect," in The IEEE International Symposium on Personal, Indoor and Mobile Radio Communications, vol. 1, pp. 172-176, 2002. Article (CrossRef Link)

[28] G. Bianchi, "Performance analysis of the IEEE 802.11 distributed coordination function," IEEE Journal on Selected Areas in Communications, vol. 18, no. 3, pp. 535-547, 2000. Article (CrossRef Link) 
[29] E. Ziouva and T. Antonakopoulos, "CSMA/CA performance under high traffic conditions: throughput and delay analysis," Computer Communications, vol. 25, no. 3, pp. 313-321, 2002. Article (CrossRef Link)

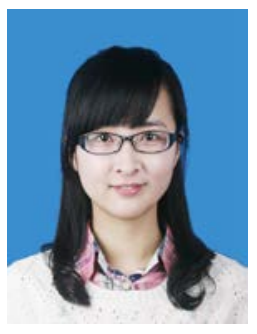

Haiwei Zuo received the B.S. degree in Information and Communication Engineering from China University of Mining and Technology in 2012. She is currently pursuing the $\mathrm{Ph} . \mathrm{D}$. degree at School of Information and Control Engineering, China University of Mining and Technology. Her research interests include IBFD communication and wireless networks.

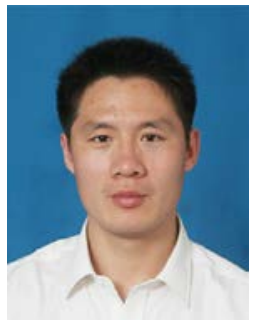

Yanjing Sun is a professor in School of Information and Control Engineering, China University of Mining and Technology since July 2012. He received the Ph.D. degree in Information and Communication Engineering from China University of Mining and Technology in 2008. His current research interests include IBFD communication, Embedded real-time system, Wireless sensor networks, Cyber-physical system and so on.

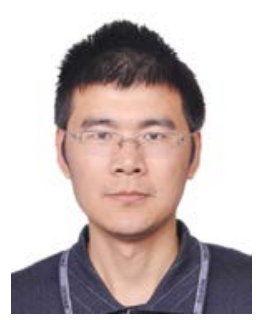

Song $\mathbf{L i}$ is an associate Professor in School of Information and Control Engineering, China University of Mining and Technology. He received the Ph.D. degree in signal and information processing from Beijing University of Posts and Telecommunications in 2012. His current research interests are full duplex communication, cyber-physical system and cooperative communication.

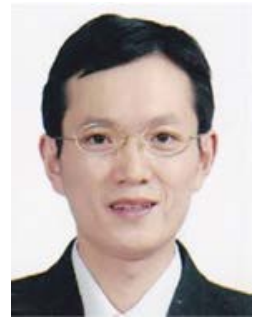

Qiang Ni is a professor and the Head of the Communication Systems Group with InfoLab21, School of Computing and Communications, Lancaster University, Lancaster, U.K. He received the B.Sc., M.Sc., and Ph.D. degrees from the Huazhong University of Science and Technology, China, all in engineering. He has authored over 180 papers in international journals and conferences. His current research interests are future generation communications and networking, including heterogeneous networks, CRN systems, 5G, SDN, and so on. He was an IEEE 802.11 Wireless Standard Working Group Voting Member and a Contributor to the IEEE Wireless Standards. 


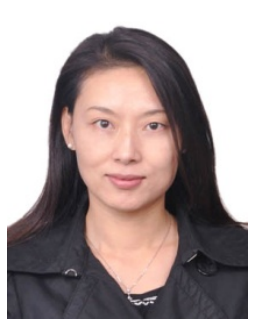

Xiaolin Wang is an associate professor in School of Information and Control Engineering, China University of Mining and Technology. She received the Ph.D degree in Resources development and planning from China University of Mining and Technology in 2012. Her current research interests are mining systems engineering, resources development and planning.

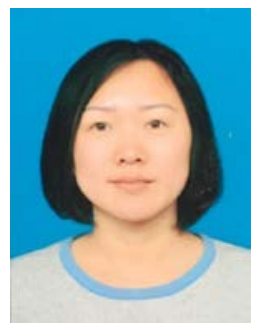

Xiaoguang Zhang is an associate professor in School of Information and Control Engineering, China University of Mining and Technology. Her current research interests are wireless communications and signal processing. 